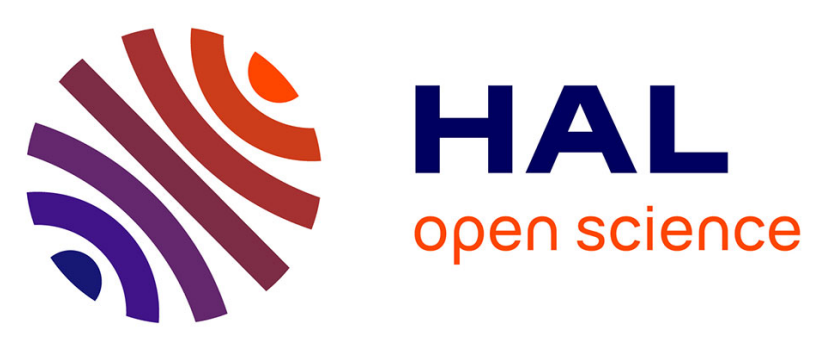

\title{
Communautés énergétiques locales, coopératives citoyennes et autoconsommation collective : formes et trajectoires en France \\ Gilles Debizet, Marta Pappalardo
}

\section{- To cite this version:}

Gilles Debizet, Marta Pappalardo. Communautés énergétiques locales, coopératives citoyennes et autoconsommation collective : formes et trajectoires en France. Flux - Cahiers scientifiques internationaux Réseaux et territoires, 2021, Communautés énergétiques locales, coopératives citoyennes et autoconsommation collective : formes et trajectoires en France, 126, pp.1-13. 10.3917/flux1.126.0001 . hal-03554278

\author{
HAL Id: hal-03554278 \\ https://hal.science/hal-03554278
}

Submitted on 16 Feb 2022

HAL is a multi-disciplinary open access archive for the deposit and dissemination of scientific research documents, whether they are published or not. The documents may come from teaching and research institutions in France or abroad, or from public or private research centers.
L'archive ouverte pluridisciplinaire HAL, est destinée au dépôt et à la diffusion de documents scientifiques de niveau recherche, publiés ou non, émanant des établissements d'enseignement et de recherche français ou étrangers, des laboratoires publics ou privés. 


\title{
Communautés énergétiques locales, coopératives citoyennes et autoconsommation collective : formes et trajectoires en France
}

\author{
Gilles Debizet, Marta Pappalardo
}

Univ. Grenoble Alpes, CNRS, Sciences Po Grenoble*, Pacte, 38000 Grenoble, France

* School of Political Studies Univ. Grenoble Alpes

\section{Les communautés énergétiques : un objet multiple dans un champ de recherche récent}

Les recherches récentes, à l'échelle française et internationale, témoignent l'intérêt pour la question des communautés énergétiques, et plus largement pour les modes d'organisation des acteurs autour de la production et de la consommation d'énergies renouvelables à l'échelle locale.

Ce numéro spécial de Flux, consacré aux communautés énergétiques locales, a pour ambition de rendre compte de la diversité des recherches récentes sur cet objet polysémique et polymorphe, à l'aide d'une mise en perspective de la littérature internationale sur les communautés énergétiques et de quatre contributions de chercheurs travaillant sur ces questions, à partir de différentes disciplines. Les quatre articles sont issus d'un travail de sélection de propositions formulées par les auteurs à la suite de leur participation au cycle de webinaires " Communautés énergétiques, autoproduction, autoconsommation : cadrages, pratiques et outils », organisé par le CDP Eco-SESA de l'Université Grenoble Alpes et le Puca, de juin à octobre 2020. Comme présenté en détail dans le compte-rendu en fin de ce numéro, les sessions du colloque ont traité des thématiques interdisciplinaires impliquant des chercheurs, mais également des acteurs institutionnels et des professionnels de l'énergie.

Depuis le début des années 2010, le modèle des communautés énergétiques a suscité l'engouement des acteurs, notamment en raison de la possibilité de participation des citoyens aux processus de délibération collectifs. Dans ce sens, cet objectif sociétal et politique s'entremêle aux valeurs environnementales, qui visent notamment une baisse des consommations d'énergie et un impact moins important sur la planète, mais surtout s'insèrent dans une tendance de sortie des logiques marchandes traditionnelles. Nombre d'acteurs associent notamment les communautés énergétiques à une idée de proximité spatiale et, implicitement, de relations interpersonnelles entre ses membres. Depuis juillet 2021, le cadre réglementaire français évolue, notamment par l'entrée en vigueur en des ordonnances (publiées en mars 2021) transposant les directives européennes RED II et IEMD ${ }^{1}$, qui définissent les règles applicables aux différents modèles de communautés énergétiques, aux garanties d'origine ainsi qu'à l'encadrement du stockage de l'électricité2.

Au-delà du cadrage réglementaire, notre définition de communauté énergétique rend compte de cette diversité d'approches et d'analyses : nous entendons par communauté énergétique un regroupement de personnes physiques ou morales activement impliquées dans un projet de production et/ou de consommation d'énergie renouvelable. Ainsi, la communauté est un objet élastique, où les représentations des acteurs ont autant de poids que les caractéristiques objectives des dispositifs : le sentiment de faire partie d'une communauté, la mise en place de formes de gouvernance horizontales, l'imbrication entre installations techniques et espaces, ou encore l'ancrage local, contribuent à façonner les communautés.

1 «La Directive Energies Renouvelables (Directive (UE) 2018/2001 du Parlement européen et du Conseil du 11 décembre 2018 relative à la promotion de l'utilisation de l'énergie produite à partir de sources renouvelables) et la Directive Electricité (Directive (UE) 2019/944 du Parlement européen et du Conseil du 5 juin 2019 concernant des règles communes pour le marché intérieur de l'électricité et modifiant la directive 2012/27/UE) du Clean Energy Package, déclinant cet objectif, ont en particulier donné lieu à l'introduction des concepts de communautés d'énergie renouvelable et de communautés énergétiques citoyennes.» Source: https://www.smartgridscre.fr/encyclopedie/les-communautes-energetiques-locales/au-dela-de-lautoconsommation-concept-decommunautes-energetiques [consulté le 11/04/2021].

2 Source : https://www.bureauveritas.fr/magazine/marche-de-lelectricite-et-promotion-des-energiesrenouvelables-transposition-de-directives [consulté le 26/06/2021]. 
Cette définition, large et polysémique, s'insère dans un panorama scientifique international reconnaissant cette diversité et les aspirations soulignées, par différentes disciplines, à des formes de transversalité et de collaboration.

\section{Débats scientifiques, en France et à l'international}

La littérature sur les communautés énergétiques révèle des différences majeures : l'expression communauté énergétique peut définir des collectifs de quelques participants jusqu'à plusieurs milliers de membres. Concernant la dimension spatiale, une communauté énergétique peut être contenue dans un seul bâtiment ou s'étendre sur de vastes territoires, être portée par des collectifs très informels jusqu'à des autorités publiques ou encore des réseaux sociaux digitaux.

Dans la diversité des configurations, la littérature internationale sur les communautés énergétiques se concentre principalement sur la dimension organisationnelle et sur la portée politique des expérimentations. Selon les cas étudiés, les cultures de recherche, les pays où sont réalisés les travaux scientifiques, la caractérisation des projets énergétiques locaux varie. Comme présenté dans l'article d'Aubert et Souami dans ce numéro spécial, si en Allemagne on parle plutôt d'énergie citoyenne (Radtke, 2013), ou d'initiatives énergétiques locales (Blanchet, 2015; Hoppe et al, 2015), la littérature anglo-saxonne s'intéresse plus spécifiquement à la communauté et à son fonctionnement, surtout quand cette dernière est mise en place par des acteurs de la société civile. Ainsi, la littérature sur les energy communities porte essentiellement sur les communautés énergétiques dites citoyennes ou grassroots (Seyfang et al, 2013 ; van der Waal et al, 2018), et à leurs formes d'organisation interne. L'attention pour les dynamiques organisationnelles, voire d'institutionnalisation des communautés énergétiques (Seyfang, Haxeltine, 2012; Wirth, 2014), est confirmée dans plusieurs travaux sur les communautés énergétiques au Royaume-Uni, dont une grande part porte sur les processus et les effets de l'implication des citoyens dans les projets (Walker, Devine-Wright, 2008).

Le caractère ambigu et élastique de la notion de communauté est reconnu par les auteurs anglophones (Hicks, Ison, 2018). Cela viendrait d'une acception positive collectivement acceptée de la notion même de community en langue anglaise, renvoyant à des connotations fortes d'empowerment des citoyens, de solidarité et de collaboration locale. Cette vision positive de la communauté se complexifie dans le contexte de la recherche francophone, où la notion de communauté peut renvoyer à la représentation négative de communautarisme. A ce propos, Yalçin-Riollet, Garabuau-Moussaoui et Szuba (2014) soulignent comment la culture linguistique française conduit à penser les communautés plutôt comme des espaces de repli et d'entre-soi, en contraste avec les valeurs de la République.

Dans les travaux de matrice anglo-saxonne, il apparaît une forme de surfocalisation sur la communauté (Aubert et Souami, dans ce numéro). Ces analyses socio-politiques méritent cependant d'être complexifiées par l'attention à des facteurs autres que la dimension organisationnelle. Le travail comparatif de Brummer (2018) met en évidence que de nombreux auteurs définissent les communautés énergétiques en fonction du sujet de leur recherche. Ainsi, si plusieurs auteurs, se concentrent sur les aspects sociaux, comme la participation de la société civile ou l'organisation des acteurs, d'autres définissent les communautés énergétiques en fonction d'aspects technologiques, comme la production d'énergie renouvelable ou une installation décentralisée (Acosta et al, 2018).

En France, les recherches sur les communautés énergétiques sont plutôt récentes, mais il existe une littérature affirmée autour de la territorialisation de l'énergie et sur l'organisation des acteurs du secteur énergétique, contribuant à l'émergence de trois débats scientifiques majeurs : les formes démocratiques des communautés, les dimensions territoriales en prenant comme point de départ les infrastructures et, les mutations des modèles économiques et de l'organisation de la filière énergétique.

Les recherches sur les systèmes de délibération internes aux communautés (Pappalardo, 2021; Maître, dans ce numéro), font état de nombre d'expérimentations dans la gouvernance des collectifs, sortant du modèle de la démocratie représentative pour aller vers des formes plus horizontales comme la sociocratie ou l'holacratie. Selon ces approches, le caractère distinctif de la communauté énergétique ne résiderait pas tant dans le type d'énergie renouvelable mis en place, mais dans l'expérimentation de modes de gouvernance. Ainsi, si les communautés énergétiques sous la forme de coopératives de production ou de fourniture sont les premières à avoir connu un déploiement important dans les territoires (Fontaine, 2019), les communautés de production et consommation in situ s'affirment actuellement comme des espaces d'expérimentation politique par la réorganisation des collectifs (Rumpala, 2013). Cette organisation se révèle profondément imbriquée dans les espaces où se déploient les pratiques de consommation de l'énergie (Pappalardo, Debizet, 2020).

Le rapport entre le réseau et ses modèles organisationnels alternatifs a fait également émerger plusieurs notions dans le champ de la géographie et de l'urbanisme à commencer par celle de post-réseau (Coutard, Rutherford, 2009, 2011) d'une transition en cours, du modèle traditionnel du grand réseau centralisé, vers des 
logiques construites autour de la dimension locale et celle de territoires énergétiques mis en œuvre à l'échelle de la ville, du quartier, du bâtiment (Souami, 2009) dont les systèmes énergétiques sont constitués d'un ensemble de noeuds socio-énergétiques (Debizet, 2013). La notion d'assemblage socio-énergétique a ainsi été mobilisée pour analyser des gouvernances énergétiques et urbaines en quête d'énergies renouvelables ou fatales (Debizet et al, 2016; Hampikian, 2017 ; Aubert, 2020), soulignant le rôle d'intermédiaires énergétiques (Tabourdeau, Debizet, 2017) dans le sillage de l'école française de la proximité (Bahers, Durand, 2017). La notion d'autonomie énergétique pointe des espaces de construction de nouveaux rapports aux ressources et de nouvelles formes d'organisation (Lopez et al, 2019).

Enfin, des travaux français issus de la sociologie se sont intéressés à l'analyse des formes économiques des communautés énergétiques, souvent à travers l'analyse de communautés productrices d'énergie ou de projets coopératifs locaux. Par les formes organisationnelles de la coopérative, de la société d'économie mixte ou encore de l'association, le local - considéré comme un espace déploiement des énergies renouvelables - impose une adaptation du projet énergétique (Dobigny, 2012; Nadaï, 2015 ; Fontaine, 2021). Ces recherches mettent en évidence les processus transcalaires, faits de tâtonnements et de prise de risques, qui confèrent à ces initiatives une dimension politique en opposition à la politique énergétique nationale (Cointe, 2016). Ces recherches interrogent le pouvoir transformatif des communautés énergétiques, voire la mise en place d'une démocratie énergétique. Par le contrôle des infrastructures énergétiques, la participation citoyenne et la distribution locale des bénéfices, les communautés énergétiques s'affirment en tant qu'espaces alternatifs aux modèles socio-économiques et marchands (Wokuri, 2021) : ces expériences sont cependant fortement conditionnées par les systèmes politiques et économiques du pays d'émergence (Wokuri, 2019).

A partir de ce panorama, il est possible de constater le peu d'attention aux dynamiques urbaines et territoriales en tant que variable structurante de l'action des communautés énergétiques. Certaines recherches identifient l'espace en tant qu'indicateur de classification des communautés énergétiques au sein d'exercices taxonomiques. A titre d'exemple, Heiskanen et al (2010) font la distinction entre : les communautés géographiquement locales, les communautés sectorielles, les communautés d'intérêt et les communautés virtuelles. Stefano Moroni et al (2019) proposent une taxonomie des communautés énergétiques qui introduit la variable territoriale dans leur caractérisation. Ainsi, ils construisent une matrice à quatre cellules : la première distinction est faite entre les communautés place-based, autrement dit construites à partir d'une dimension locale affichée et opérante, et les non place-based. La deuxième distinction porte sur les ambitions de la communauté, qui peuvent être à objectif unique (notamment autour de la fonction énergie) ou à objectifs multiples (single-purpose ou multi-purpose). Ces travaux taxonomiques permettent de réaliser des typologies de communautés énergétiques où la dimension spatiale n'est pas entendue comme un réceptacle des opportunités politiques ou d'intérêts à l'échelle locale, mais en tant qu'élément opérant dans la structuration des groupes et dans leurs capacités d'action.

Dans ce contexte, les communautés énergétiques se révèlent aujourd'hui en tant que modèles d'une spatialité renouvelée autour de l'énergie (Dubois, Kébir, 2021), qui se décline dans différentes acceptions et qui s'inscrit dans une tradition de remise en cause du modèle dominant centralisé. Du désir d'autonomie, qui fait naître des projets citoyens qui revendiquent la localité comme instrument de soutenabilité (Brusadelli, 2016), à l'utilisation de la communauté énergétique en tant qu'outil dans la discussion autour de la production urbaine (Ramirez-Cobo et al, 2021), la mise en commun d'acteurs autour de la consommation et de la production d'énergie convoque non seulement l'espace en tant que lieu où situer les dispositifs énergétiques, mais également des réflexions autour de la localité en tant qu'ensemble de valeurs (Hérault et al, 2012 ; Martin, Upham, 2016 ; Perlaviciute et al, 2018), comme la traçabilité de l'énergie, la sortie des grandes infrastructures de distribution, ou encore la proximité géographique entre producteurs et consommateurs. Dans ce sens, les communautés énergétiques sont des objets profondément incarnés dans un espace local, autrement dit dans une réflexion sur l'espace qui invite à sortir des conceptions traditionnelles de l'énergie et de sa gestion.

\section{Les contributions de ce numéro spécial}

Dans le cadre des débats récents sur les communautés énergétiques locales, en particulier dans le contexte français et des pays dits électrifiés (Europe, Amérique du Nord, Asie), les articles sélectionnés pour ce numéro spécial rendent compte des différentes dynamiques liées à trois aspects significatifs de l'émergence et de la mise en œuvre des communautés énergétiques locales, à savoir l'imbrication dans l'espace, l'organisation de la gouvernance et le rapport aux régulations nationales et supranationales.

La dimension urbaine fait l'objet de l'analyse proposée par la contribution de Flora Aubert et Taoufik Souami. Par une recherche questionnant avant tout la définition et la mobilisation de la notion de communauté énergétique dans la littérature internationale, les auteurs soulignent la nécessité de sortir d'une vision de cet objet, mouvant et 
élastique, focalisée sur les rapports socio-politiques entre les acteurs. Leur analyse propose de mobiliser les cadres analytiques des systèmes énergétiques locaux pour intégrer la matérialité des dispositifs techniques et des espaces urbains dans l'analyse des dynamiques conduisant à l'émergence et à la mise en œuvre de communautés énergétiques locales. A travers l'étude de cas de trois communautés d'autoconsommation collective électrique situées en France, en Allemagne et au Royaume-Uni - les auteurs proposent une analyse de l'action de ces collectifs par le prisme de la fabrique urbaine, à savoir l'ensemble d'outils, de mécanismes et de logiques impliquant des acteurs humains et non-humains dans la mise en œuvre des communautés en tant que systèmes socio-énergétiques urbains.

Quels qu'en soient les acteurs, la notion de communauté énergétique demande donc un passage d'une dimension individuelle à une dimension collective : l'organisation des acteurs autour d'une ressource - l'énergie renouvelable - et leur réflexion sur un développement de la consommation et de la production énergétique qui soit alternatif, voire innovant, sont au cœur des recherches des contributeurs de ce numéro. Plus particulièrement, dans son article, Arnaud Assié analyse les dynamiques du faire autrement, à savoir la recherche de nouvelles manières de s'organiser pour produire et consommer de la ressource et de nouvelles réglementations qui soient adaptées à ces configurations inédites. Par son analyse des épreuves rencontrées par les projets citoyens d'énergies renouvelables, l'auteur montre comment les visions idéalisées des porteurs de ces projets se heurtent à un fonctionnement transcalaire et à la nécessité de mettre en place des outils de gouvernance. L'étude du fonctionnement de l'association Energie Partagée à l'échelle nationale, mais également des projets locaux se revendiquant comme appartenant à cette mouvance d'énergie citoyenne, montrent des imbrications complexes entre des acteurs et des dispositifs qui obligent les acteurs à composer avec la nécessité de créer des réseaux d'échange et de faire appel à des compétences cognitives d'appui. Ces interrogations nous invitent à questionner le lien avec les réseaux : quelle alternative est possible, dans un contexte dominé par une centralisation économique, politique et spatiale des grands réseaux de distribution? Dans ce sens, les différentes contributions de ce numéro relatent de la volonté des acteurs de l'énergie, soient-ils privés, publics ou citoyens, d'expérimenter des voies de sortie d'une centralisation accrue de la production et de la gestion des énergies, ainsi que de leurs infrastructures de gestion.

Dans ce sens, l'analyse des modes de délibération, à savoir les modalités par lesquelles les acteurs se rassemblent, négocient, voire parviennent à une décision commune sur les aspects techniques, spatiaux et organisationnelles de la communauté, fait l'objet de plusieurs recherches sur les communautés énergétiques, dont la contribution de Rémi Maître. Par son étude de cas sur Enercoop, fournisseur coopératif d'électricité EnR à l'échelle nationale et de ses déclinaisons régionales - en particulier Enercoop Midi-Pyrénées (EMIP) - l'auteur interroge le fonctionnement de ces collectifs portant en même temps les ambitions paradoxales de baisse des consommations et de fourniture d'énergie renouvelable. Organisés selon trois principes : un fonctionnement par SCIC (Société Coopérative d'Intérêt Collectif), une déconcentration du niveau national au niveau régional, et une configuration organisationnelle autour de l'holacratie, Enercoop construit une offre alternative au marché traditionnel de l'énergie, non seulement du point de vue économique, mais plus encore dans le sens d'une participation active de la part des membres du réseau. Après une présentation de l'organisation d'EMIP, qui organise son fonctionnement de manière prépondérante autour de la Décision Par Consentement (DPC), l'article mobilise la sociologie praxéologique pour analyser la gouvernance et les modes de circulation de l'information des membres de la coopérative.

Enfin, dans la contribution de Thibaut Fonteneau, la communauté énergétique devient un instrument d'une organisation politico-économique alternative, mettant à jour les controverses liées à la régulation nationale du marché de distribution de l'énergie. Plus particulièrement, à travers le cas d'étude de trois collectifs d'autoconsommation collective électrique en France, l'auteur analyse les controverses issues des demandes d'adaptation des tarifs d'utilisation des réseaux (TURPE). Son analyse révèle la contradiction transcalaire entre les principes de solidarité nationale, incarnés dans le réseau centralisé de distribution de l'énergie, et la dimension locale, espace d'émergence de dynamiques et d'acteurs d'une transition énergétique décentralisée.

Le numéro est complété par un entretien avec Didier Laffaille, Secrétaire général du Comité de prospective de la Commission de régulation de l'énergie (CRE) par Gilles Debizet. Consacré essentiellement aux dispositifs légaux d'autoconsommation individuelle et d'autoconsommation collective d'électricité, cet entretien met en lumière l'attention à l'économie du réseau et à la forme d'équité incarnée par le principe d'uniformité du tarif d'accès à l'électricité quel que soit le lieu de production ou de consommation, discutée dans l'article de T. Fonteneau. Enfin, le compte-rendu du cycle de webinaires international «Communautés énergétiques, autoproduction, autoconsommation : cadrages, pratiques et outils », réalisé par Marta Pappalardo, permet d'élargir les questions évoquées dans les contributions susnommées et d'alimenter des pistes de réflexion transdisciplinaires. 


\section{Émergence et déploiement des communautés énergétiques locales en France}

Les quatre contributions relatent d'une période particulière qui a fait l'objet de nombreuses propositions de communications : le passage de l'émergence au déploiement des communautés énergétiques. Les articles publiés dans ce numéro et le compte-rendu du cycle de webinaires mettent en exergue des « épreuves » que traversent les projets de communauté énergétique ainsi que des stratégies de déploiement mises en place par les différents acteurs. Entre initiatives citoyennes, action publique locale et dispositions gouvernementales, plusieurs processus d'institutionnalisation des communautés énergétiques locales s'esquissent. L'analyse transversale des textes de ce numéro spécial met en exergue des différences sensibles et autant de questions saillantes : l'alternative locale au modèle électrique dominant, la relation au local et les trajectoires institutionnelles et de déploiement.

\section{La communauté locale comme contre-modèle au régime dominant?}

Instaurer un contre-modèle aux systèmes énergétiques dominants constitue une motivation forte et commune des personnes impliquées dans des opérations communautaires de production d'énergies renouvelables. La vision d'un modèle centralisé et technocratique - dont le nucléaire français est l'archétype - fédère les oppositions.

Au-delà de cette opposition au modèle centralisé, des colorations diffèrent selon l'objet de la communauté. Dans son enquête sur le réseau Enercoop, Rémi Maitre souligne la réaction au poids prépondérant des grandes entreprises « capitalistiques » ou « spéculatives » - nationales et internationales - dans la production des énergies renouvelables. Des dérives des certificats de garantie d'origine organisés dans le cadre d'un marché européen sont dénoncées pour justifier l'activité de la coopérative française de fourniture d'électricité d'origine renouvelable.

Dans l'état de l'art scientifique, Arnaud Assié pointe plus précisément la logique - que nous appelons - extractiviste de ces entreprises, c'est à dire qui consiste à valoriser une ressource à l'extérieur de son territoire d'extraction sans que ce dernier n'en perçoive un bénéfice. Les pratiques sur le terrain sont ainsi parfois qualifiées de prédatrices, d'inéquitables, et même, de coloniales ${ }^{3}$ (Yalçin-Riollet et al, cit. ; Poize, Rudinger, 2014). En contrepoint de ces pratiques, le mouvement Energie partagée présente les retombées sur le territoire comme un objectif intrinsèque à chaque opération. D'une part, les investisseurs coopérateurs résidant généralement dans le bassin de vie tirent un revenu financier qui est généralement réinvesti dans d'autres projets locaux. D'autre part, les coopérateurs soutiennent des activités sociales et environnementales du territoire (Assié). La charte d'Energie Partagée explicite ces deux valeurs : un ancrage local et une démarche non spéculative.

Cette charte énonce deux autres valeurs : une exigence écologique et une gouvernance ouverte de type démocratique (Assié). Cette dernière se traduit par le le choix, fait par Énergie Partagée comme par Enercoop, du statut de Scic - Société Coopérative d'Intérêt Collectif - qui permet à des personnes physiques ou morales de différents statuts de prendre part de façon ouverte et démocratique $(1$ personne $=1$ voix $)$ aux décisions de la coopérative. L'objectif commun est de démocratiser l'énergie (Maître), là-aussi en contrepoint des producteurs dominants de l'électricité - fossile, fissile ou renouvelable - considérés comme technocratiques ou capitalistiques.

Les deux autres articles de ce numéro portent un point de vue sensiblement moins affirmatif sur l'objectif de mise en place d'un contre-modèle. Thibaut Fonteneau présente les opérations d'autoconsommation collective comme des opportunités saisies par des acteurs locaux tels que les Communes et les bailleurs sociaux pour faire bénéficier leurs administrés, respectivement leurs locataires, d'une énergie peu onéreuse, verte et locale. Il est vrai que ces deux types d'acteurs agissent essentiellement localement et ont une légitimité peu discutée à cette échelle. Autrement dit, ils occupent déjà une place bien spécifique dans le système institutionnel français : inutile de se positionner en contre-modèle même si cela n'exclut une lutte pour l'élargissement de leurs moyens et prérogatives (Poupeau, Boutaud, 2021).

Somme toute, la nouvelle implication des collectivités locales dans les énergies renouvelables serait pour les acteurs locaux un élargissement sectoriel de la fabrique urbaine, estiment Flora Aubert et Taoufik Souami en étudiant des territoires où sont mis en œuvre des projets d'autoconsommation collective ou de production/injection par des coopératives citoyennes. Cette implication résulte selon eux d'une dynamique locale, en l'occurrence la fabrique urbaine : elle ne se réduit pas à leur capacité à dépasser les contraintes à la valorisation des ressources situées.

3 L'expression, rapportée par Yalçin-Riollet et Garabuau-Moussaoui, est d'un fonctionnaire territorial jouant un rôle clé dans la démarche d'autonomie énergétique du canton breton du Mené. 
Retenons deux points à ce stade. Contrairement au mouvement coopératif de l'énergie, l'utilisation du dispositif d'autoconsommation collective ne semble pas nécessiter l'adhésion à un contre-modèle - non-spéculatif, démocratique et écologique - par rapport au modèle dominant. Si les actions des coopératives énergétiques s'inscrivent en opposition au modèle énergétique, elles sont, du point de vue de la fabrique urbaine et territoriale, considérées comme un élargissement thématique. En quoi les articles révèlent la contribution des communautés énergétiques locales à cette fabrique ?

\section{L'énergie communautaire à l'épreuve du local : passage obligé versus émanation}

« Les projets dits citoyens se distinguent par la prise en compte d'enjeux territoriaux » affirme Arnaud Assié. La régionalisation d'Enercoop répond à un objectif de proximité avec les adhérents, les abonnés ainsi qu'avec les producteurs d'énergie renouvelable (Maître). Les coopératives citoyennes visent une forme de re-territorialisation de l'énergie. Les deux auteurs décrivent précisément les conséquences de cette position sur la structuration des réseaux Enercoop et Energie Partagée mais peu sur l'opérationnalité de la re-territorialisation.

Par des analyses sociotechniques fines, Flora Aubert, Taoufik Souami et Thibaut Fonteneau révèlent des modalités de mise en œuvre de l'autoconsommation collective. Ils soulignent ainsi la dépendance à la matérialité du réseau électrique, du réseau viaire et du maillage foncier. Soumises à des statuts de propriétés spécifiques, ces trois objets sont au cœur de la fabrique urbaine. Les auteurs montrent aussi l'importance de la métrique spatiale définissant le périmètre maximum des opérations d'autoconsommation collective. Cette métrique s'avère «actante » dans le ciblage des autoconsommateurs collectifs (Aubert et Souami). Elle a fait l'objet de débats et d'ajustements dans plusieurs scènes impliquant les représentants des collectivités locales, des législateurs nationaux et de la haute administration de l'Etat en charge de la politique énergétique nationale (Fonteneau).

L'essaimage par proximité spatiale est une autre dimension de la territorialisation. Le principe de réinvestissement des bénéfices dans de nouveaux projets localisés dans le périmètre d'action des coopératives locales d'Energie Partagée (Assié) tout comme le soutien à des projets de production de la part d'EMIP (coopérative régionale d'Enercoop agissant en Midi-Pyrénées) territorialise la ressource financière indispensable au déploiement de projets renouvelables. Outre le financement, est aussi ressource territoriale - au sens de Gumuchian et Pecqueur (2007) - l'expertise en montage de projet et en animation de communautés énergétiques locales, d'abord apportée par les fondateurs bénévoles et désormais par les salariés des coopératives régionales de Enercoop (Maître) et ceux des réseaux régionaux rassemblant les coopératives adhérentes d'Energie Partagée (Assié). Enfin, la réussite d'opérations démontre la faisabilité et les bénéfices des projets et des communautés énergétiques locales, ce qui suscite et conforte les initiatives de proche en proche à la façon d'Hagerstrand (1967) et selon la théorie de la diffusion des innovations de Rogers (2003).

Somme toute, autant que les collectivités locales elles-mêmes et d'autres acteurs ancrés dans le territoire (par exemple l'université de Nottingham ou l'entreprise locale de distribution d'énergie de Saerbeck selon Aubert et Souami), les mouvements coopératifs Enercoop et Energie Partagée contribuent doublement à la territorialisation de l'énergie. En effet, ces derniers d'une part apportent des ressources immatérielles dans leurs territoires d'action et, d'autre part, constituent une organisation transcalaire sensiblement moins descendante que celle du régime électrique dominant. Le basculement du point de vue - du système électrique à celui du territoire - renvoie à la notion d'opérateur territorial proposée par Durand et Landel (2015) : les organisations de production d'énergie renouvelable seraient des opérateurs que les acteurs du territoire sollicitent pour valoriser les ressources énergétiques ancrées. Ainsi, les systèmes énergétiques territorialisés pourraient être considérés comme des autonomies connectées entre eux par des réseaux régulés (ADEME, 2019; Debizet, 2019).

\section{Deux niches et trajectoires institutionnelles : coopératives versus autoconsommation collective}

Comme affirmé précédemment, les opérations d'autoconsommation collective sont portées par des acteurs le plus souvent déjà établis localement, qui élargissent leur activité au champ de l'énergie électrique alors que les coopératives de production et de consommation d'énergie renouvelable sont des organisations nouvelles. Les trajectoires de l'autoconsommation collective et des coopératives énergétiques s'avèrent sensiblement différentes. Pour comprendre ces trajectoires, mobilisons l'approche multi-niveaux (Geels, 2004) qui est au cœur du courant de recherche des Sustainability Transition Studies.

Cette approche distingue trois temporalités : primo, le paysage qui correspond à des dimensions culturelles et à des valeurs qui pour l'essentiel évoluent lentement sauf choc conjoncturel; secundo le régime sociotechnique constitué par un ensemble cohérent d'infrastructures, d'organisations et de règles qui les relient dont la stabilité permet à certaines d'entre les premières d'occuper une place dominante ; tertio, les niches au sein desquelles des innovations vont émerger et se déployer de façon limitée avant que leur propagation ne bouscule le régime 
sociotechnique. Dans certains cas, la généralisation d'un bouquet d'innovations peut suffisamment déstabiliser le régime sociotechnique pour le transformer en profondeur et/ou faire émerger un nouveau régime sociotechnique.

La libéralisation du marché du gaz et de l'électricité corollaire à la construction européenne et la conscience générale de la nécessité de limiter le changement climatique ont récemment (eu égard à l'histoire plus que centenaire des réseaux électriques) émergé au niveau paysage. Le régime sociotechnique de l'électricité en France résulte de la nationalisation, à la sortie de la seconde guerre mondiale, des entreprises locales et régionales privées d'électricité. La création de l'entreprise publique nationale Electricité de France a permis la construction de puissantes centrales hydroélectriques puis nucléaires. Ce régime électrique très fortement centralisé à l'échelle nationale a été amendé au tournant des années 2000 par la libéralisation de la production et de la fourniture et, plus récemment, par l'instauration de niches dans lesquelles éclosent des projets d'énergie renouvelable portées par des communautés énergétiques. C'est au niveau niches que se distinguent les opérations d'autoconsommation collective et celles portées par des coopératives : dérogation à la règle d'interdiction de vente ou de cession d'électricité en dehors des fournisseurs agréés pour les premières, espaces marchands protégés pour la production d'énergie renouvelable pour les secondes.

Dans le premier cas, l'Etat - dans la diversité de ses institutions - a défini depuis 2019 un dispositif dérogatoire en encadrant précisément les modalités d'échange d'électricité (Fonteneau). Il instaure le principe d'une entité intermédiaire contractualisant séparément avec le(s) producteur(s) et les consommateurs volontaires, définit ses responsabilités vis-à-vis du gestionnaire de réseau et fixe finalement une distance maximale entre les lieux de production et de consommation. Cette condition spatiale sort de facto l'autoconsommation collective du joug des mécanismes de marché basés sur le principe d'une multiplicité d'offreurs et d'indépendance des demandeurs les uns vis-à-vis des autres. Ainsi, le dispositif appelle des délibérations portant sur - voire réunissant - le(s) producteur(s) et l'ensemble des consommateurs adhérents.

Dans le second cas, les coopératives se saisissent d'incitations (prix d'achat garanti par l'Etat puis bonus supplémentaire au prix moyen du marché) et de dispositions (exemple des garanties d'origine renouvelable) que l'Etat a instaurées depuis le début des années 2000 afin de créer un espace marchand protégé permettant le développement des installations d'énergie renouvelable en France. Autrement dit, les coopératives jouent pleinement avec les mécanismes de marché : Enercoop achète de l'énergie renouvelable et la revend à des consommateurs/adhérents; Energie Partagée produit de l'énergie renouvelable et l'injecte généralement intégralement dans le réseau en la revendant à des fournisseurs, dont Enercoop. Ils se positionnent de facto - le premier comme fournisseur, le second comme producteur - sur le marché concurrentiel national tout en revendiquant une visée locale (Assié à propos de Energie Partagée) ou désormais régionale (Maître à propos de Enercoop).

\section{Imbrication entre économies d'échelles, professionnalisation et organisation}

Si la rentabilité n'est pas l'objectif central des communautés énergétiques, elle s'avère bien souvent nécessaire à la concrétisation des installations de production d'énergie renouvelable, du moins aux subventions près. Si chaque opération est spécifique du fait de son ancrage géographique et politique, des facteurs et des dynamiques leurs sont communes. Ainsi, la recherche d'économie d'échelle et de baisse des coûts prend des formes spécifiques au type de communautés énergétiques.

La coopérative Enercoop ayant fait le choix d'une croissance rapide, elle s'est régionalisée afin d'attirer davantage d'abonnés mais elle conserve au niveau national la responsabilité de fournisseur - et donc la relation client -, la relation avec les producteurs à qui elle achète l'énergie renouvelable et l'action de représentation et de plaidoyer auprès des institutions nationales et européennes (Maître). Dans un mouvement inverse, la nouvelle fédération Energie Partagée a progressivement fédéré des associations et coopératives locales. Ce faisant, la fédération nationale dispose d'un pool d'expertise technique, économique et juridique qui élabore des guides et des formations à l'intention des coopératives locales et intervient dans des actions de plaidoyer uni- ou multilatérales auprès des autorités nationales. Les projets «locaux» de très grande envergure peuvent aussi bénéficier de financements de la coopérative nationale, contrôlée par des organisations fondatrices de la fédération (Assié). Même si leur trajectoire transcalaire est opposée (du national au régional pour Enercoop, du local au national pour Énergie Partagée), les deux réseaux coopératifs organisent des économies d'échelle via leurs composantes nationales.

Comparativement à ces mouvements coopératifs transcalaires, les porteurs d'opérations d'autoconsommation collective menées en France paraissent isolés. Les personnes morales sont - à ce stade de déploiement - spécifiques à chaque opération. La faible taille (induite par l'encadrement de la distance métrique) ne laisse pas espérer des 
économies d'échelle à court ou moyen terme. C'est donc par la congruence d'objectifs que ces opérations généralement non rentables - aboutissent : chacune satisfait plusieurs objectifs et mobilise des ressources propres de son porteur (Aubert et Souami) : bailleurs sociaux et municipalités profitent de leur connaissance du terrain et de leur légitimité pour recruter des auto-consommateurs. A l'inverse des mouvements coopératifs, ces organisations ne portent une opération d'autoconsommation collective que de façon occasionnelle ; n'ayant pas les moyens de développer une expertise technique et juridique en leur sein, elles recourent à des entités extérieures : il s'agit souvent de bureau d'études ou d'entreprises de services énergétiques (Fonteneau, Aubert et Souami).

\section{Concilier déploiement et financement du réseau?}

Des fédérations nationales rassemblant ces entreprises et des députés sollicités par des élus locaux mènent des actions de plaidoyer pour l'élargissement du périmètre spatial et pour la réduction de la taxe finançant le réseau (Fonteneau). Si par leur taille et leur nombre actuel, les opérations d'autoconsommation collective représentent aujourd'hui une quantité négligeable dans le financement du réseau, le régulateur national de l'énergie se montre cependant prudent, comme l'atteste l'entretien avec Didier Laffaille, Secrétaire du comité prospective de la Commission de Régulation de l'Energie, dans ce numéro spécial. Les coûts d'investissement et de fonctionnement du réseau électrique public découlent de sa capacité à faire transiter des flux instantanés (ce qui correspond aux moments de pointe de soutirage par les abonnés) et peu du volume transité (qui eux correspondent au cumul des soutirages sur une longue période). Or, le financement du réseau électrique public est essentiellement assis sur les volumes consommés; en conséquence, une baisse du taux de taxe pour les flux collectivement consommés nécessiterait une augmentation du taux pour les flux allogènes (ceux qui ne relèvent pas du dispositif d'autoconsommation collective). Cela remettrait en cause le principe dit du «timbre-poste » en vigueur depuis 1973, c'est-à-dire de l'uniformité sur le territoire national du prix d'acheminement de l'électricité. Dans cette conjoncture, l'autoconsommation collective apparaît dans une impasse économique : même régime de taxe mais très faible possibilité d'économies d'échelles comparativement aux productions allogènes. Cela explique pourquoi ces opérations sont généralement portées par des entités existantes qui, d'une part, produisent principalement pour leur autoconsommation individuelle (qui est de facto exemptée de taxes) et, d'autre part, tirent des bénéfices nonmarchand de l'autoconsommation collective : la satisfaction d'administrés et de locataires, le renforcement de l'économie locale ou la mise en pratique de valeurs écologiques. Cumulée à la complexité de mise œuvre, l'impasse économique explique aussi le faible nombre d'opérations d'autoconsommation collective: une cinquantaine en 2021.

En revanche, les opérations portées par des coopératives citoyennes connaissent un essor important en France depuis quelques années (Assié). Dans le sillage des directives européennes introduisant la notion de communautés énergétiques, Enercoop et Energie Partagée plaident - avec le Comité de Liaison des Énergies Renouvelables pour l'introduction d'un critère d'implication citoyenne dans les appels à projets soutenant les installations d'énergie renouvelable ; l'objectif est de faire bénéficier les projets citoyens d'un bonus plus important que celui attribué aux projets portés par des entreprises privées classiques, c'est-à-dire celles dont la rémunération du capital est la finalité centrale. Les interventions de François Ménard, Cyril Martin de la Garde et Thomas Rüdinger lors de la table ronde de clôture du cycle de webinaires "Communautés énergétiques, autoproduction, autoconsommation : cadrages, pratiques et outils $»^{4}$ esquissent des modalités retenues par l'ordonnance de mars 2021 : un financement par des personnes physiques localisées, proximité de l'installation de production, statut coopératif.

\section{Conclusion : débats et perspectives politiques en France}

Il convient tout d'abord d'attirer l'attention du lecteur sur le fait que les quatre articles et l'entretien avec Didier Laffaille concernent quasi-exclusivement le vecteur électrique alors que la notion de communauté énergétique n'exclut pas des échanges de gaz, de chaleur ou de bois, chacun de ces vecteurs pouvant être issu d'énergies renouvelables. Il existe pourtant des dizaines de milliers d'immeubles résidentiels qui mobilisent des énergies renouvelables captées in situ pour alimenter leur système de chauffage collectif; il existe aussi des centaines de réseaux publics de chaleur. Ces communautés énergétiques «de facto» suscitent des controverses locales (analysées dans les thèses doctorales de Tardieu, 2015 ; Blanchard 2018 ; Hampikian cit. ; Balaye, 2019 ; Aubert cit.) mais ne semblent pas être abordées dans les débats nationaux de la transition énergétique. Il est vrai qu'aucun agrément national n'est nécessaire pour vendre de la chaleur ou du bois et que leurs lieux d'extraction et de transformation sont très nombreux par comparaison aux unités de production d'électricité. C'est peut-être parce

4 Voir compte-rendu du cycle de webinaires en fin de ce numéro spécial. 
que les régimes sociotechniques du bois-énergie et de la chaleur sont peu centralisés qu'ils ne stimulent pas de controverses nationales, ni le contre-modèle de communauté énergétique.

Revenons aux enseignements de l'analyse transversale des articles. Somme toute, le large déploiement d'opérations de production d'électricité renouvelables, portées selon une démarche de type communautés énergétiques, requiert une institutionnalisation conjointement ascendante et descendante. Les six textes rassemblés dans ce numéro témoignent de deux trajectoires d'institutionnalisation bien distinctes :

- La construction d'un mouvement coopératif transcalaire et la prise en compte de ses spécificités dans la régulation des marchés de l'énergie : des coopératives citoyennes locales de production progressivement organisées en réseau national et une coopérative nationale de fourniture d'électricité désormais partiellement déconcentrée en région ont saisi la libéralisation de l'énergie et l'instauration d'une niche protégée du marché favorisant le déploiement d'énergies renouvelables. Remplacer la phrase par : Vingt après la libéralisation de l'électricité et la création des premières coopératives, les régulations européennes et nationales reconnaissent leur spécificité citoyenne et locale ;

- L'instauration - à la suite d'un débat parlementaire - d'un dispositif dérogatoire à l'exclusivité de vente d'électricité accordée aux fournisseurs de marché (agréés par l'Etat) dont se sont saisi une diversité d'acteurs des territoires et d'entreprises de services et d'équipement : introduite par la loi sur la Transition énergétique et la Croissance verte de 2015, l'idée d'élargissement au collectif de l'autoconsommation individuelle s'est incarnée dans un dispositif réglementaire en 2018 ; depuis, ce dispositif a fait l'objet d'ajustements au gré des retours d'expériences effectués par les quelques organisations capables de les porter dans des scènes nationales mais il n'offre toujours pas de viabilité économique.

Complémentaires entre eux, les modèles économiques et organisationnels développés par les deux réseaux coopératifs s'avèrent antagoniques avec le modèle d'autoconsommation collective que d'autres acteurs mettent en œuvre sur le terrain et plaident au niveau régional et national. Le moment de verrouillage - au sens de Flichy (1995) - d'un statut de «communauté énergétique citoyenne » au sein des régulations de l'énergie approche ; le décret qui suivra l'ordonnance de mars 2021 pourrait être le prélude d'un large déploiement des coopératives de production/injection si le gouvernement ouvre les vannes du financement. En revanche, le devenir de l'autoconsommation collective paraît très incertain puisque son passage à l'échelle suppose l'inflexion - et donc la remise en cause - du principe de tarification « timbre-poste ».

Il résulte de cette histoire récente trois modèles de communauté énergétique basés sur le vecteur électrique. 1) le modèle coopérative citoyenne participe au fonctionnement du marché (national et européen) tout en organisant un entrelacs d'interdépendances entre des coopératives de fourniture et des coopératives de production/injection à différentes échelles spatiales ; 2) l'autoconsommation collective extrait du marché de l'électricité la part qui lie collectivement un ou des producteurs avec des consommateurs spatialement proches tout en contribuant au financement du réseau ; 3) un modèle peu visible mais bien présent, l'autoconsommation aval-compteur peut s'avérer communautaire de facto pour certains usages (Pappalardo, Debizet, cit.). C'est aussi un modèle d'autoconsommation aval-compteur public qui est généralement en vigueur pour les productions collectives de chaleur renouvelable. Dans ces deux derniers cas, les organisations immobilières classiques remplissent souvent elles-mêmes les fonctions de délibération et de supervision des flux.

Le relation entre réseau et territoire est réinterrogée par la notion - et les réalités afférentes - de communauté énergétique locale. Observer cette relation depuis le territoire - et pas seulement depuis le réseau - met en lumière le poids et l'hétérogénéité des organisations collectives existantes. À l'heure où il apparaît que la transition sera conjointement écologique et solidaire, c'est aussi sous l'angle de la justice et de la démocratie qu'il convient de ré-explorer la relation entre réseau et territoire. Pour ne citer que deux exemples, si la justice procédurale (qui inspire la décision par consentement relatée par R. Maître) conduit à décliner des scènes de délibération relatives au partage des énergies produites à différentes échelles, le postulat d'un égal accès à l'électricité - quels que soient le lieu et le « collectif » auquel est rattachée une unité de consommation - réduit de facto les effets de délibérations aux échelles territoriales intermédiaires. Les nouveaux fronts de recherche de la justice énergétique (van Bommel, Höffken, 2021) et de la démocratie énergétique (Szulecki, Overland, 2020) auront à prendre en compte les échelons de planification et de régulation et à investiguer la gestion des énergies au sein des communautés, auto-déclarées ou désignées, utilisant - plus ou moins à l'écart des mécanismes de marché - des ressources communes. 


\section{Remerciements}

Ce travail a bénéficié du soutien du Cross-Disciplinary Program Eco-SESA Smart Energies in Districts recevant des financements de l'Agence Nationale de la Recherche, au titre du programme " Investissements d'avenir » portant la référence ANR-15-IDEX-02, de l'ADEME (recherche RETHINE) et de la Région Auvergne-RhôneAlpes (recherche OREBE).

\section{Bibliographie}

Acosta, C., Ortega, M., Bunsen, T., Prasad Koirala, B., Ghorbani, A., 2018, Facilitating Energy Transition through Energy Commons: An Application of Socio-Ecological Systems Framework for Integrated Community Energy Systems, Sustainability, N 10, p. 366.

Ademe, 2019, Systèmes énergétiques territorialisés : interactions multivecteurs, feuille de route stratégique, Montrouge : Ademe, ISBN 9791029712487.

Aubert F., 2020, Communautés énergétiques » et fabrique urbaine ordinaire : analyses croisées Allemagne, France, Royaume-Uni, Thèse de doctorat en Aménagement de l'espace, Urbanisme sous la direction de Taoufik Souami et Xavier Bonnaud, Ecole des Ponts ParisTech, Université de Paris-Est. http://www.theses.fr/2020PESC2016

Bahers J.-B., Durand M., 2017, Le retour de la proximité ! Quelles implications pour les services urbains en réseau ?, Flux, N 109-110, p. 1-8. DOI : 10.3917/flux1.109.0001. URL : https://www.cairn.info/revue-flux-20173-page-1.htm

Balaye F., 2019, La construction territoriale de la gouvernance des réseaux de distribution d'électricité : le cas des Métropoles de Brest et Grenoble, Thèse de doctorat en Aménagement et urbanisme sous la direction de Bernard Pecqueur et Gilles Debizet, Université Grenoble Alpes. http://www.theses.fr/2019GREAH037

Blanchard G., 2018, Comment La Maîtrise d'ouvrage Urbaine Conçoit-Elle Les Choix d'aménagement ? : Élaboration et Assemblage Des Choix Énergétiques à Bordeaux Euratlantique, Thèse de doctorat en Aménagement de l'espace, Urbanisme sous la direction de Taoufik Souami, de l'université Paris Est. http://www.theses.fr/2018PESC1156.

Blanchard Ph., 2010, Les médias et l'agenda de l'électronucléaire en France. 1970-2000, Thèse de doctorat en Science politique, sous la direction de Jacques Gerstlé, Université Paris 9.

Blanchet T., 2015, Struggle over energy transition in Berlin: how do grassroots initiatives affect local energy policy-making?, Energy Policy, vol. 78, pp. 246-254.

Brummer V., 2018, Community energy - benefits and barriers: A comparative literature review of Community Energy in the UK, Germany and the USA, the benefits it provides for society and the barriers it faces, Renewable and Sustainable Energy Reviews, vol. 94, pp. 187-196, ISSN 1364-0321, https://doi.org/10.1016/j.rser.2018.06.013.

Brusadelli N., Lemay M., Martell Y., 2016, L’espace contemporain des « alternatives ». Un révélateur des recompositions des classes moyennes?, Savoir/Agir, 2016/4, N³8, pp. 13-20.

Cointe B., 2016, Le tarif d'achat photovoltaïque comme outil d'innovation territoriale : l'exemple des Fermes de Figeac, VertigO - la revue électronique en sciences de l'environnement [En ligne], vol. 16, N ${ }^{\circ} 1 . \mathrm{URL}^{\mathrm{K}}$ http://journals.openedition.org/vertigo/17040 ; DOI : https://doi.org/10.4000/vertigo.17040

Coutard O., Rutherford J,. 2011, The Rise of Post-Networked Cities in Europe? Recombining Infrastructural Ecological and Urban Transformations in Low Carbon Transitions'. In Cities and Low Carbon Transitions, 10725. Routledge Studies in Human Geography. Bulkeley, Castan Broto, Hodson, Marvin.

Coutard O., Rutherford J., 2009, Les réseaux transformés par leurs marges : développement et ambivalence des techniques « décentralisées », Flux, 2009/2-3, N 76-77, pp. 6-13. DOI : 10.3917/flux.076.0006. URL : https://www.cairn.info/revue-flux1-2009-2-page-6.htm

Debizet G., 2013, Les coordinations trans-scalaires de l'énergie en ville La notion de nœud socio-énergétique. Colloque Futurs Urbains, Jan 2013, Marne-La-Vallée, France. 〈halshs-00827180〉 
Debizet G., 2019, Repenser fiabilité et solidarité au prisme des autonomies connectées, in : Lopez F., Pellegrino M., Coutard O. (sous la direction de), Les territoires de l'autonomie énergétique. Espaces, échelles et politiques, ISTE Editions, pp. 87-102.

Debizet G., Tabourdeau A., Gauthier C., Menanteau Ph., 2016, Spatial processes in urban energy transitions: considering an assemblage of Socio-Energetic Nodes, Journal of Cleaner Production, vol. 134, Part A, pp. 330341, https://doi.org/10.1016/j.jclepro.2016.02.140.

Dobigny, L, 2012, Chapitre 8. Produire et échanger localement son énergie. Dynamiques et solidarités à l'œuvre dans les communes rurales ", in: Papy F. (sous la direction de), Nouveaux rapports à la nature dans les campagnes, Éditions Quæ, pp. 139-152.

Dubois J., Kebir L., 2021, Éditorial. Transition énergétique : le retour des lieux, Espaces et sociétés, 2021/1, N 182, pp. 9-14. DOI : 10.3917/esp.182.0009. URL : https://www.cairn.info/revue-espaces-et-societes-2021-1-page9.htm.

Durand L., Landel P.-A., 2015, L'émergence de l'opérateur territorial de l'énergie, Géocarrefour [En ligne], $\mathrm{N}^{\circ}$ 90/4. URL : http://journals.openedition.org/geocarrefour/9980 ; DOI : https://doi.org/10.4000/geocarrefour.9980.

Flichy P., 1995, L’innovation Technique, Récents Développements En Sciences Sociales Vers Une Nouvelle Théorie de l'innovation, Paris :. Editions La Découvertee. Sciences et Société.

Fontaine A., 2019, L'essor des coopératives énergétiques citoyennes, Multitudes, 2019/4, № 77, pp. 88-93. DOI : 10.3917/mult.077.0088. URL : https://www.cairn.info/revue-multitudes-2019-4-page-88.htm

Fontaine A., 2021, Expérimenter une transition énergétique coopérative : épreuves et innovations territoriales collectives autour d'un projet de « centrale photovoltaïque villageoise » (Rhône-Alpes, France), Natures Sciences Sociétés, N²9, pp. 36-45. DOI : https://doi.org/10.1051/nss/2021027

Geels F. W., 2004, From Sectoral Systems of Innovation to Socio-Technical Systems: Insights about Dynamics and Change from Sociology and Institutional Theory'. Research Policy, $\mathrm{N}^{\circ} 33$ (6-7):, pp. $897-920$. https://doi.org/10.1016/j.respol.2004.01.015

Gumuchian H., Pecqueur B. (sous la direction de), 2007, La ressource territoriale. Paris: Economica.

Hagerstrand T., 1967,. Innovation Diffusion as a Spatial Process, Chicago: . University of Chicago Press.

Hampikian Z., 2017, De la distribution aux synergies ? : Circulations locales d'énergie et transformations des processus de mise en réseau de la ville, Thèse de doctorat en Aménagement de l'espace, Urbanisme, sous la direction de Taoufik Souami, Université Paris-Est. https://pastel.archives-ouvertes.fr/tel-01586025/document

Heiskanen E., M. Johnson, S. Robinson, E. Vadovics, M. Saastamoinen, 2010, Low-carbon communities as a context for individual behavioural change, Energy Policy, vol. 38, ํㅜ 12, pp. 7586-7595.

Hérault-Fournier C., Merle A., Prigent-Simonin A. H., 2012, Comment les consommateurs perçoivent-ils la proximité à l'égard d'un circuit court alimentaire ?, Management \& Avenir, 2012/3, $\mathrm{N}^{\circ}$ 53, pp. 16-33. DOI: 10.3917/mav.053.0016. URL: https://www.cairn.info/revue-management-et-avenir-2012-3-page-16.htm

Hicks J., Ison N., 2018, An exploration of the boundaries of 'community' in community renewable energy projects: Navigating between motivations and context, Energy Policy, № 113, pp. 523-534.

Hoppe T., Graf A., Warbroek B., Lammers I. et Lepping I., 2015, Local Governments Supporting Local Energy Initiatives: Lessons from the Best Practices of Saerbeck (Germany) and Lochem (The Netherlands), Sustainability, vol. 7, $\mathrm{N}^{\circ} 2$, pp. 1900-1931.

Lopez F., Pellegrino M., Coutard O. (sous la direction de), 2019, Les territoires de l'autonomie énergétique. Espaces, échelles et politiques, ISTE Editions.

Martin C. J., Upham P., 2016, Grassroots social innovation and the mobilisation of values in collaborative consumption: a conceptual model, Journal of Cleaner Production, vol. 134, Part A, pp. 204-213, https://doi.org/10.1016/j.jclepro.2015.04.062.

Moroni S., Alberti V., Antoniucci V. Bisello A., 2019, Energy communities in the transition to a low-carbon future: A taxonomical approach and some policy dilemmas, Journal of Environmental Management, vol. 236, pp. 45-53. 
Nadaï A., Labussière O., Debourdeau A., Régnier Y., Cointe, B., Dobigny L., 2015, French policy localism: surfing on 'Positive Energie Territories' (Tepos), Energy Policy, № 78, pp. 281-91.

Pappalardo M., 2021, La gouvernance des communautés énergétiques, entre pratiques de l'espace et dynamiques de pouvoir, Espaces et sociétés, 2021/1, $\mathrm{N}^{\circ}$ 182, pp. 55-71. DOI : 10.3917/esp.182.0055. URL : https://www.cairn.info/revue-espaces-et-societes-2021-1-page-55.htm

Pappalardo M., Debizet G., 2020, Understanding the governance of innovative energy sharing in multi-dwelling buildings through a spatial analysis of consumption practices, Global Transitions, vol. 2, pp. 221-229, https://doi.org/10.1016/j.glt.2020.09.001.

Perlaviciute G., Steg L., Contzen N., Roeser S., Huijts N., 2018, Emotional Responses to Energy Projects: Insights for Responsible Decision Making in a Sustainable Energy Transition, Sustainability, MDPI, Open Access Journal, vol. 10(7), pp. 1-12.

Poupeau F-M., Boutaud B., 2021, La transition énergétique, un nouveau laboratoire de l'action publique locale ?. Pouvoirs Locaux : les cahiers de la décentralisation, 2021, vol. 1I, $\mathrm{N}^{\circ}$ (119), pp.28-36.

Radtke J., 2013, Bürgerenergie in Deutschland - ein Modell für Partizipation?, in: Radtke J., Hennig B. (eds.), Die deutsche Energiewende nach Fukushima. Marburg: Metropolis-Verlag, pp. 139-182.

Ramirez-Cobo I., Tribout S., Debizet G., 2021, Territoires d'énergie, territoires à projet. Articulations et dépendances entre conceptions urbaine et énergétique, Espaces et sociétés, 2021/1, N 182, pp. 73-91. DOI : 10.3917/esp.182.0073. URL : https://www.cairn.info/revue-espaces-et-societes-2021-1-page-73.htm

Rogers E., 2003., Diffusion of Innovations,. 5th ed., New York: Free Press.,

Rumpala Y., 2013, Formes alternatives de production énergétique et reconfigurations politiques. La sociologie des énergies alternatives comme étude des potentialités de réorganisation du collectif, Flux, 2013/2, N 92, pp. 47-61. DOI : 10.3917/flux.092.0047. URL : https://www.cairn.info/revue-flux1-2013-2-page-47.htm

Seyfang G., Haxeltine A., 2012, Growing grassroots innovations: exploring the role of community-based initiatives in governing sustainable energy transitions, Environment and Planning C: Government and Policy, vol. 30, pp. 381 - 400, doi:10.1068/c10222.

Seyfang G., Park J. J., Smith A., 2013, A thousand flowers blooming? An examination of community energy in the UK, Energy Policy, vol. 61, pp. 977-989, https://doi.org/10.1016/j.enpol.2013.06.030.

Souami T., 2009, Conceptions et représentations du territoire énergétique dans les quartiers durables, Flux, 2009/23, $\mathrm{N}^{\circ}$ 76-77, pp. 71-81. DOI : 10.3917/flux.076.0071. URL : https://www.cairn.info/revue-flux1-2009-2-page$\underline{71 . h t m}$

Szulecki K., Overland I., 2020, Energy democracy as a process, an outcome and a goal: A conceptual review, Energy Research \& Social Science, vol. 69, https://doi.org/10.1016/j.erss.2020.101768.

Tabourdeau A., Debizet G., 2017, Concilier ressources in situ et grands réseaux : une lecture des proximités par la notion de nœud socio-énergétique, Flux, 2017/3-4, N 109-110, pp. 87-101. DOI : 10.3917/flux1.109.0087. URL : https://www.cairn.info/revue-flux-2017-3-page-87.htm

Tardieu C., 2015, Transition énergétique dans les projets urbains : conditions de mise en æeuvre. Analyse des cas Paris Rive Gauche, Clichy-Batignolles et Paris Nord Est, Thèse de doctorat en Aménagement et urbanisme, sous la direction de Olivier Blanpain, Morgane Colombert et Youssef Diab, Université Lille 1. http://www.theses.fr/2015LIL10024

van Bommel N., Höffken J. I., 2021, Energy justice within, between and beyond European community energy initiatives: A review, Energy Research \& Social Science, vol. 79, https://doi.org/10.1016/j.erss.2021.102157.

van der Waal E. C., Windt H. J. van der Oost E. C. J., 2018, « How Local Energy Initiatives Develop Technological Innovations: Growing an Actor Network », Sustainability, vol. 10, N 12, pp. 45-77.

Walker G., Devine-Wright P., 2008, Community renewable energy: What should it mean?, Energy policy, vol. 36, $\mathrm{N}^{\circ} 2$, pp. 497-500.

Wirth S., 2014, Communities matter: Institutional preconditions for community renewable energy, Energy Policy, $\mathrm{N}^{\circ} 70$, pp. 236-246. 
Wokuri P., 2019, Participation citoyenne et régimes de politiques publiques : nouvelle donne ou donne inchangée ? Le cas des projets coopératifs d'énergie renouvelable au Danemark et en France, Lien social et Politiques, $\mathrm{N}^{\circ}$ 82, pp. 158-180. https://doi.org/10.7202/1061881ar

Wokuri P., 2021, Community Energy in the United Kingdom: beyond or between the Market and the State?, Revue Française de Civilisation Britannique [Online], No 26-2. URL: http://journals.openedition.org/rfcb/7976. DOI: https://doi.org/10.4000/rfcb.7976

Yalçın-Riollet M., Garabuau-Moussaoui I., Szuba M., 2014, Energy autonomy in Le Mené: A French case of grassroots innovation, Energy Policy, vol. 69, pp. 347-355. 